OPEN ACCESS

Edited by:

Natascha D. Wagner, University of Göttingen, Germany

Reviewed by: Angela Jean McDonnell, Chicago Botanic Garden, United States

Kyong-Sook Chung, Jungwon University, South Korea

*Correspondence: Mingli Yan ymljack@126.com Meiliang Zhou zhoumeiliang@caas.cn

Specialty section: This article was submitted to Plant Systematics and Evolution,

a section of the journal

Frontiers in Plant Science

Received: 20 February 2020 Accepted: 30 June 2020 Published: 16 July 2020

Citation:

Cheng C, Fan Y, Tang Y, Zhang $K$,

Joshi DC, Jha R, Janovská $D$, Meglič V, Yan M and Zhou M (2020)

Fagopyrum esculentum ssp. ancestrale-A Hybrid Species Between Diploid F. cymosum and F. esculentum. Front. Plant Sci. 11:1073. doi: $10.3389 /$ fpls.2020.01073

\section{Fagopyrum esculentum ssp. ancestrale-A Hybrid Species Between Diploid $F$. cymosum and $F$. esculentum}

\author{
Cheng Cheng ${ }^{1,2}$, Yu Fan ${ }^{1}$, Yu Tang ${ }^{3}$, Kaixuan Zhang ${ }^{1}$, Dinesh C. Joshi ${ }^{4}$, Rintu Jha ${ }^{1}$, \\ Dagmar Janovská ${ }^{5}$, Vladimir Meglič ${ }^{6}$, Mingli Yan $^{2 *}$ and Meiliang Zhou ${ }^{1 *}$

\begin{abstract}
${ }^{1}$ Institute of Crop Sciences, Chinese Academy of Agricultural Sciences, Beijing, China, ${ }^{2}$ School of Life Sciences, Hunan University of Science and Technology, Xiangtan, China, ${ }^{3}$ Department of Tourism, Sichuan Tourism University, Chengdu, China, ${ }^{4}$ Indian Council of Agricultural Research-Vivekananda Institute of Hill Agriculture, Almora, India, ${ }^{5}$ Gene Bank, Crop Research Institute, Prague, Czechia, ${ }^{6}$ Crop Science Department, Agricultural Institute of Slovenia, Ljubljana, Slovenia
\end{abstract}

Fagopyrum cymosum is considered as most probable wild ancestor of cultivated buckwheat. However, the evolutionary route from F. cymosum to $F$. esculentum remains to be deciphered. We hypothesized that a hybrid species exists in natural habitats between diploid $F$. cymosum and $F$. esculentum. The aim of this research was to determine the phylogenetic position of $F$. esculentum ssp. ancestrale and to provide new thoughts on buckwheat evolution. Different methodologies including evaluation of morphological traits, determination of secondary metabolites, fluorescence in situ hybridization (FISH), comparative chloroplast genomics, and molecular markers were deployed to determine the phylogenetic relationship of $F$. esculentum ssp. ancestrale with $F$. cymosum and F. esculentum. The ambiguity observed in morphological pattern of genetic variation in three species revealed that $F$. esculentum ssp. ancestrale is closely related to $F$. cymosum and $F$. esculentum. Flavonoid analysis revealed that $F$. esculentum ssp. ancestrale is closely related to $F$. esculentum. Comparative chloroplast genome analysis further supported the close proximity of $F$. esculentum ssp. ancestrale with $F$. esculentum. Additionally, molecular marker analysis revealed that $F$. esculentum ssp. ancestrale exhibits co-dominance with the bands amplified by $F$. cymosum and $F$. esculentum. These finding provided supporting evidence in favor of the hypothesis that F. esculentum ssp. ancestrale is a hybrid species between F. cymosum to F. esculentum, which was probably originated by spontaneous hybridization under natural conditions.

Keywords: buckwheat, chloroplast genome, Fagopyrum esculentum ssp. ancestrale, molecular markers, phylogenetics

\section{INTRODUCTION}

Buckwheat (Fagopyrum spp.) originated in southwestern China is one of the oldest domesticated crops from Asia. Out of the 26 known species of genus Fagopyrum, that includes diploids $(2 \mathrm{n}=2 \mathrm{x}=$ 16) and tetraploids $(2 \mathrm{n}=4 \mathrm{x}=32)$, F. esculentum (common buckwheat) and F. tataricum (tartary buckwheat) are the two cultivated species (Joshi et al., 2020). Due to the alterable (ordered) position 
of Fagopyrum in family Polygonaceae, it was difficult to deduce the evolutionary patterns and phylogenetic relationships of Fagopyrum species for a long time (Ohnishi, 2016). In order to understand the complex phylogenetic relationships and domestication events, Fagopyrum species are classified into two groups based on kernel morphology: the big achene group (cymosum group) and the small achene group (urophyllum group) (Ohnishi and Matsuoka, 1996). Except for kernel morphology, two diploid varieties $F$. cymosum var. pilus and $F$. cymosum var. megaspartanium of the large achene group were very similar to the two cultivated species in many aspects and were considered the potential ancestors of common buckwheat (F. esculentum) and tartary buckwheat (F. tataricum) respectively (Steward, 1930; Ohnishi, 2016). However, reports based on morphological cladistics, isozyme analysis, and DNA polymorphism suggested that $F$. cymosum is not the direct ancestor of buckwheat; it is only distantly related to $F$. tataricum and F. esculentum (Ohnishi and Matsuoka, 1996; Kadyrova et al., 2010; Zhou et al., 2012).

To further resolve the issue of buckwheat evolution, Ohnishi (1991; 1998) proposed two wild species; F. esculentum ssp. ancestrale from Yunan province and F. tataricum ssp. potanini from Sichuan province of China as probable ancestors of $F$. esculentum and F. tataricum respectively. The two candidate species were considered as wild progenitors of cultivated species because of their differences in dormancy and shattering habit that are mainly used to make a distinction between cultivated species and its close wild relatives (Ohnishi and Matsuoka, 1996).

During our germplasm expedition undertaken from the year 2016-2018, we found a dense distribution of F. esculentum ssp. ancestrale populations in Yunnan province of China. Upon examining the morphological pattern of genetic variation, it was observed that wild populations of F. esculentum ssp. ancestrale are quite similar to $F$. cymosum and F. esculentum raising the hypothesis that $F$. esculentum ssp. ancestrale is a hybrid species between F. cymosum and F. esculentum. However, due to their strong dependency on environment and stage specific expression, morphological parameters are not the most reliable and informational entities in taxonomic and phylogenetic studies. Therefore, in the present paper, we describe the findings of subsequent experiments based on secondary metabolites (flavonoid) analysis, fluorescence in situ hybridization (FISH), comparative chloroplast genomics and DNA polymorphism performed to define the phylogenetic relationships of $F$. esculentum ssp. ancestrale with $F$. cymosum and F. esculentum. The FISH with total nuclear and ribosomal DNA as probes is an easy and robust method of molecular cytogenetics to physically map and compare buckwheat genomes (Walling et al., 2013), which may provide visible karyotypes of three buckwheat species under study. Moreover, chloroplast genomes are considered as a potentially useful tool in plant evolutionary studies, because of the non-meiotic and mostly uniparental inheritance of genes. The comparative analysis of chloroplast genomes indicated that the Fagopyrum possesses some unique structural features including the InDel markers that can define the evolutionary relationships of different species
(Logacheva et al., 2008; Cho et al., 2015). Finally, robust microsatellite markers rendered them as the most reliable and informational entities to complement morphological descriptors in buckwheat taxonomic and phylogenetic studies. This study contributes to new insights of buckwheat evolution.

\section{MATERIALS AND METHODS}

\section{Plant Materials}

A total of 22 accessions belonging to 15 Fagopyrum species comprising thirteen wild buckwheat species including F. esculentum ssp. ancestrale and two cultivated species (F. esculentum and F. tataricum) were used in the study (Table S1). These accessions were collected from different regions of Yunnan, Sichuan, and Beijing in China during the year 2016 to 2018. Out of the total 15 species enlisted in Table S1, F. esculentum ssp. ancestrale, F. cymosum-Luojishan and cultivated F. esculentum-Yuqiao were used for chromatography, FISH, chloroplast genome analysis, and molecular marker analysis, while the remaining twelve species were utilized for phylogenetic analysis using three molecular markers: ITS, $m a t \mathrm{~K}$, and $t r n H-p s b A$.

\section{Chromatography Analysis}

The buckwheat seeds dried to consistent weight were pulverized and sieved through a 40 mesh. The powdered sample $(0.2 \mathrm{~g})$ mixed with $10 \mathrm{ml} 80 \%$ methanol in an Erlenmeyer flask was incubated in an ultrasonic cleaner at $40 \mathrm{khz}$ and $45^{\circ} \mathrm{C}$ for $30 \mathrm{~min}$ and then passed the $0.45 \mu \mathrm{m}$ filter. The test sample was the mixture of $1 \mathrm{ml}$ filtrate, $2 \mathrm{ml}$ aluminum trichloride $\left(0.1 \mathrm{~mol} \mathrm{~L}^{-1}\right)$, and $3 \mathrm{ml}$ potassium acetic acid $\left(1 \mathrm{~mol} \mathrm{~L}^{-1}\right.$ and $4 \mathrm{ml}$ methanol $(80 \%, v / v)$. High performance liquid chromatography (HPLC) was used to measure the content of rutin and quercetin (Zhang et al., 2018). The total flavonoid content was determined by the aluminum trichloride method (colorimetric wavelength $420 \mathrm{~nm}$ ) (Li et al., 2008).

\section{Fluorescence In Situ Hybridization (FISH) Analysis}

The buckwheat seeds were submerged in water for $6 \mathrm{~h}$ at room temperature and then at $4{ }^{\circ} \mathrm{C}$ for 1 day, and afterwards sprouted at a consistent temperature of $25^{\circ} \mathrm{C}$ until the root tip was $1-2 \mathrm{~cm}$ long. The root tips were treated with nitrous oxide at $10 \mathrm{~atm}$ for $2 \mathrm{~h}$ in $1.5 \mathrm{ml}$ Eppendorf tubes, incubated in 90\% acetic acid for $8 \mathrm{~min}$, and washed with ultrapure water for three times. The root tips were digested with cellulase and pectinase at $37^{\circ} \mathrm{C}$ for $90 \mathrm{~min}$ and washed with $70 \%$ ethanol for three times on ice. The dissecting needle was used to smash the root tip in $30 \mu \mathrm{l}$ of $70 \%$ ethanol, centrifuged at low speed for 30-60 s. After discarding the supernatant, re-suspend with $30 \mu \mathrm{l}$ acetic acid for $5 \mathrm{~min}$. The suspension of 5-7 $\mu \mathrm{l}$ was used to determine the karyotypes by the microscopy observation (Stebbins, 1971; Hsiao et al. 1986). The $25 \mathrm{~S}$ rDNA probe was generated by nick translation of a $2.3 \mathrm{~kb}$ ClaI subclone of the $25 \mathrm{~S}$ rDNA coding region of Arabidopsis thaliana as described by Jenkins and 
Hasterok (2007). This probe was labeled with digoxigenin-11dUTP (Roche) and used to visualize 45S rDNA loci containing the genes coding for $18 \mathrm{~S}, 5.8 \mathrm{~S}$, and $25 \mathrm{~S}$ rRNA. Pictures were captured by the OLYMPUS AX80 microscopy (Olympus Corporation, Japan) with a CCD camera (Diagnostic Instruments, USA).

\section{DNA Extraction, Sequencing, and Assembly}

The total genomic DNA was extracted from $100 \mathrm{mg}$ of fresh leaves by the modified CTAB method (Li et al., 2013). Subsequently, the total DNA was disrupted by ultrasound to produce fragments of 300-500 bp and the fragment quality was checked using Bioanalyzer 2100 (Agilent Technologies). A 400 bp DNA library was constructed using the NEB Next Ultra ${ }^{\mathrm{TM}}$ DNA Library Kit (Illumina, San Diego, California, USA). To assemble the chloroplast genome, we firstly using Hiseq $4000 \mathrm{PE}$ 150 (Illumina, San Diego, California, USA) to sequencing the library fragments, then SPAdes (Bankevich et al., 2012) was used for de novo assemblies, the contigs obtained were further screened by BLAST, after that, Sequencher 4.10 (http://www. genecodes.com) were used to marge the screened contigs, finally, Geneious 8.1 (Kearse et al., 2012) was used to compare all reads to the spliced chloroplast genome sequence to test whether the counting sequence was correct or not.

\section{Chloroplast Genome Analysis}

The chloroplast genome annotation of F. cymosum, F. esculentum and F. esculentum sp. Ancestrale was performed using DOGMA software (Wyman et al., 2004). BLASTX and BLASTN programme were utilized to search for the location of coding genes, transfer RNAs, and ribosomal RNAs. Due to its limitations, BLAST cannot annotate short exons as a result of which some exon intron regions were not well represented in $\mathrm{cp}$ genome. To overcome this, we made precise adjustments for the annotation based on other published chloroplast genome information. The circular genome maps were drawn by Organellar Genome DRAW (http://ogdraw.mpimpgolm.mpg) (Lohse et al., 2013) and edited by Adobe Illustrator CS5. The microsatellites (SSRs) were searched in cp genome using the Misa-web programme (Beier et al., 2017; https://webblast.ipkgatersleben.de/misa/). The parameter (unit size/minimum number of repeats) utilized for the search were ten repeat units for mononucleotide microsatellites, six units for dinucleotide microsatellites and five repeat units each for tri, tetra, penta, and hexanucelotide microsatellites. The cp genomes of five buckwheat species (F. cymosum, F. tataricum, F. esculentum, $F$. esculentum sp. ancestrale, F. lujojishanense) were compared using m VISTA programme (Frazer et al., 2004) to reflect the unique characteristics of species. The five chloroplast genomes were compared using MAFFT v5 software (Katoh and Standley, 2013) with the default parameter settings for the alignment process, following with manual sequence adjustment by Seal (http://tree.bio.ed.ac.uk/software/seal.html). The principle of comparison was to open the inversions that appear in the sequence to avoid erroneous data polymorphism. The LSC, SSC and IR regions of the five species were calculated using the DnaSP v5.0 software. The sequence information of the five species were presented in Table 1.

\section{Phylogenetic Analysis}

Phylogenetic analysis was performed on cp genome sequences of Fagopyrum species and other species of four closely related dicotyledonous genra (Figure 7). In this study, we used nine species belonging to Polygonaceae families, including five species of Fagopyrum (F. cymosum, F. tataricum, F. esculentum, F. esculentum sp. ancestrale, $F$. lujojishanense) for investigating the phylogenetic relationships of Fagopyrum species. The remaining four species belonging to genus Rumex or genus Rheum were used as outgroups. The chloroplast genome information and nucleotide sequence data were obtained from NCBI (Table S2). The phylogenetic tree based on a maximum likelihood method was constructed using RA x ML v7.2.8 (Stamatakis, 2006) with a bootstrap value of 1,000 .

\section{Molecular Markers Analysis}

Molecular marker analysis was performed on genomic DNA isolated from the leaves of $F$. esculentum ssp. ancestrale, $F$. esculentum and F. cymosum. The nucleotide sequences of SSR markers and PCR cycling conditions are furnished in Supplementary Table S2.

\section{Statistical Analysis}

The results of total flavonoid content determination and chromosome arm ratio measurement presented in this article were based on the average of three parallel experiments. The data were analyzed with appropriate methods with the SPSS software (SPSS v. 25.0; IBM Co., Armonk, NY, USA). The statistical treatment was performed using Student's t-test to examine the significant differences at a significance level of $P<0.01$. Data mapping was processed using Origin 8.0 software (Origin Lab vol. 9.1).

TABLE 1 | Comparison of the complete chloroplast genome contents of five Fagopyrum species.

\begin{tabular}{|c|c|c|c|c|c|}
\hline GenBank accession number & $\begin{array}{l}\text { F. cymosum } \\
\text { KY275181 }\end{array}$ & $\begin{array}{l}\text { F. luojishannse } \\
\text { KY275182 }\end{array}$ & $\begin{array}{l}\text { F. esculentum } \\
\text { MT572345 }\end{array}$ & $\begin{array}{l}\text { F. tataricum } \\
\text { KM201427 }\end{array}$ & $\begin{array}{l}\text { F. esculentum ssp. ancestrale } \\
\text { MT572344 }\end{array}$ \\
\hline Total sequence length & 159,320 & 159,265 & 159,576 & 159,272 & 159,600 \\
\hline Large signal copy (LSC) & 84,422 & 84,431 & 84,875 & 84,398 & 84,892 \\
\hline Small signal copy (SSC) & 13,264 & 13,094 & 13,331 & 13,292 & 13,334 \\
\hline Inverted repeat region (IR) & 30,817 & 30,870 & 30,685 & 30,791 & 30,687 \\
\hline Total number of genes & 114 & 114 & 113 & 114 & 113 \\
\hline
\end{tabular}




\section{RESULTS}

F. esculentum ssp. ancestrale Is Morphologically Related to F. cymosum and $F$. esculentum

Phylogenetic relationships of F. esculentum ssp. ancestrale with $F$. cymosum and F. esculentum based on morphological characters are discussed in this section. A perusal of morphological characters revealed that differences among three buckwheat species occur for plant height, leaf morphology, perianth color and fruit shape (Figure 1, Table 2). For instance, perianth of $F$. esculentum ssp. ancestrale was characterized by white color whereas; F. cymosum and $F$. esculentum possess either white or pink colored perianth. Similarly, F. esculentum ssp. ancestrale can be distinguished by the presence of long triangular shaped achenes. In contrast, $F$. cymosum and F. esculentum were characterized by ovate shaped achenes. On account of leaf morphology, growth habit, inflorescence type, and seed color, all the three species appeared to be closely related to each other and no distinction can be made among them (Table 2). The ambiguity observed in morphological traits of F. esculentum ssp. ancestrale, F. cymosum and $F$. esculentum supported our hypothesis that $F$. esculentum ssp. ancestrale is a hybrid species between F. cymosum and F. esculentum.

\section{Flavonoid Pattern Confirmed Phylogenetic Relationship of $F$. esculentum ssp. ancestrale With $F$. esculentum and F. cymosum}

The total flavonoids, rutin and quercetin content of F. esculentum ssp. ancestrale, F. cymosum and F. esculentum were determined. F. cymosum had the highest total flavonoid content $\left(17.9 \mathrm{mg} \mathrm{g}^{-1}\right)$ followed by $F$. esculentum ssp. ancestrale ( $4.6 \mathrm{mg} \mathrm{g}^{-1}$ ) and F. esculentum (2.9 $\left.\mathrm{mg} \mathrm{g}^{-1}\right)$. Likewise, significant differences were observed for the rutin content of F. cymosum $\left(14.7 \mathrm{mg} \mathrm{g}^{-1}\right)$ compared to the F. esculentum ssp. ancestrale $\left(2.9 \mathrm{mg} \mathrm{g}^{-1}\right)$ and F. esculentum $\left(0.5 \mathrm{mg} \mathrm{g}^{-1}\right)$ (Figure 2). While quercetin content was observed in trace quantity $\left(0.6 \mathrm{mg} \mathrm{g}^{-1}\right)$ in F. cymosum, it was absent in F. esculentum ssp. ancestrale and F. esculentum. Overall, it was observed that F. esculentum ssp. ancestrale is more closely related to F. esculentum compared to F. cymosum based on total flavonoids, rutin and quercetin contents.

\section{Cytogenetic Analysis Revealed Diploid Genomic Constitution of $F$. esculentum ssp. ancestrale and Its Close Proximity With $F$. esculentum}

The mitotic chromosome analysis through FISH revealed diploid $(2 \mathrm{n}=2 \mathrm{x}=16)$ genomic constitution of all the three species under investigation (Figure 3). The arm ratio of F. esculentum ssp. ancestrale and F. esculentum was from 1.033 to 1.563 and from 1.119 to 1.406 respectively, suggesting that their chromosome types were quite similar $(2 \mathrm{n}=2 \mathrm{x}=16 \mathrm{~m})$ (Levan et al., 1964). Whereas, the $F$. cymosum was characterized as $2 \mathrm{n}=2 \mathrm{x}=15 \mathrm{~m}$ $+1 \mathrm{sm}$ by the presence of fifteen metacentric chromosomes (arm ratio 1.063-1.744) and one sub-metacentric chromosome (arm ratio 1.744). The ratio of the longest to the shortest chromosome of F. cymosum, F. esculentum ssp. ancestrale and F. esculentum was $1.967,1.561$, and 2.267 .

\section{Chloroplast (cp) Genomics of $F$. esculentum and $F$. esculentum ssp. ancestrale}

The cp genome of $F$. esculentum produced a total number of 0.58 million pair-end reads with $42.63 \mathrm{~Gb}$ of clean data. The size of the complete cp genome was $159,576 \mathrm{bp}$, which displayed a typical quadripartite structure, including a pair of inverted repeat region (2IRR; 30,685 bp) separated by the large single copy (LSC; $84,875 \mathrm{bp}$ ) and small single copy (SSC; $13,331 \mathrm{bp}$ ) regions (Figure S1, Table 1). Likewise, the cp genome of F. esculentum ssp. ancestrale was represented by the size of 159,600 bp and

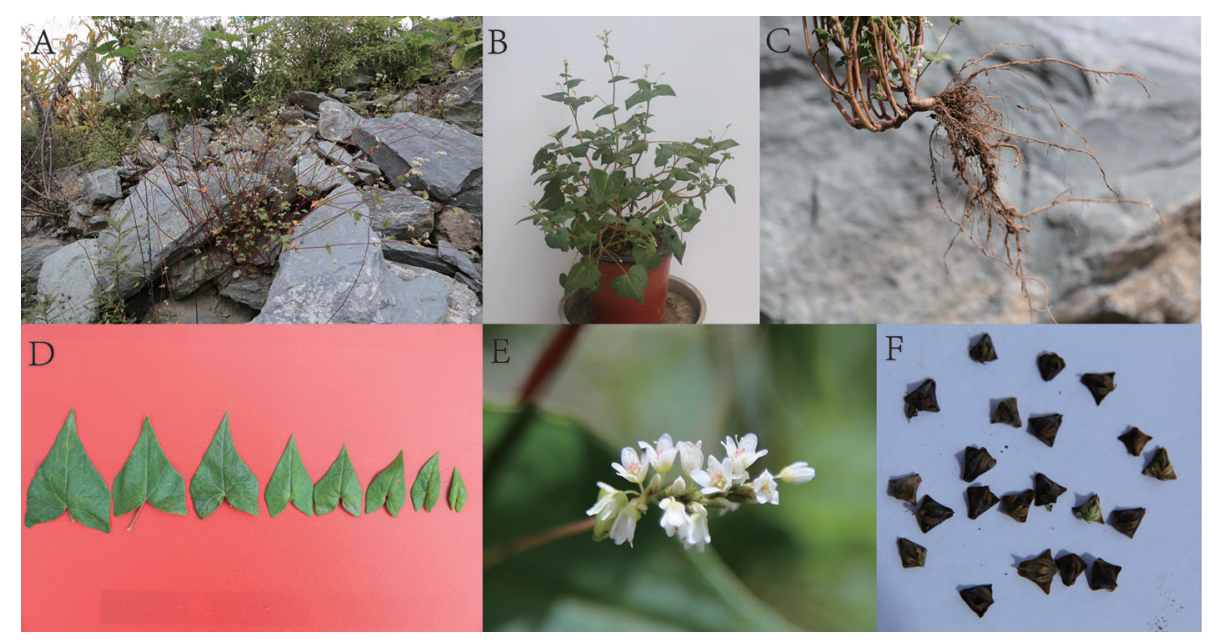

FIGURE 1 | The morphological characters of F. esculentum ssp. ancestrale. A: Habitat, B: Individual pattern, C: Root, D: Leaf, E: Flower, F: Seed. 
TABLE 2 | Morphological characters of F. cymosum, F. esculentum ssp. ancestrale and $F$. esculentum.

\begin{tabular}{|c|c|c|c|}
\hline $\begin{array}{l}\text { Index } \\
\text { Origin }\end{array}$ & $\begin{array}{l}\text { F. cymosum } \\
\text { Cultivar }\end{array}$ & $\begin{array}{l}\text { F. esculentum ssp. ancestrale } \\
\text { Wild }\end{array}$ & $\begin{array}{l}\text { F. esculentum } \\
\text { Cultivar }\end{array}$ \\
\hline Plant height (cm) & $50 \sim 200 \mathrm{~cm}$ & $50.5 \sim 154.0 \mathrm{~cm}$ & $40 \sim 80 \mathrm{~cm}$ \\
\hline Plant type & mostly erect, sometimes semi-erect & semi-erect & erect \\
\hline Stem color & green or red-brown & green or red-brown & green or red \\
\hline Leaf morphology & $\begin{array}{l}\text { Triangular, Leaf width3-11 cm, leaf length 4- } \\
12 \mathrm{~cm}\end{array}$ & $\begin{array}{l}\text { triangular, long oval and arrow shaped } \\
\text { Leaf width } 0.4 \sim 12.4 \mathrm{~cm} \text {, leaf length } 1.4 \sim 11 \\
\mathrm{~cm}\end{array}$ & $\begin{array}{l}\text { triangular or ovate triangular, Leaf width } 2-5 \mathrm{~cm} \text {, } \\
\text { leaf length } 2.5-7 \mathrm{~cm}\end{array}$ \\
\hline Petioles & $\begin{array}{l}\text { leaves in upper part of stem have no petioles } \\
\text { or short petioles }\end{array}$ & $\begin{array}{l}\text { leaves in upper part of stem have no petioles } \\
\text { or short petioles }\end{array}$ & $\begin{array}{l}\text { leaves in upper part of stem have no petioles or } \\
\text { short petioles }\end{array}$ \\
\hline Inflorescence type & Capitate, axillary and terminal & Capitate, axillary and terminal & Capitate, axillary and terminal \\
\hline $\begin{array}{l}\text { Perianth number } \\
\text { and color }\end{array}$ & 5 , white or pink & 5, white & 5 , white or pink \\
\hline Number of stamens & 8 & 8 & 8 \\
\hline Number of style & 3 & 3 & 3 \\
\hline Flower type & Homomorphic flower & Homomorphic or Heteromorphic flower & Heteromorphic flower \\
\hline
\end{tabular}

diversity

Fruit color Black-brown, no lustrous

Fruit type and Achene, Broadly ovate

Black-brown, no lustrous

Achene, Long triangular

Black-brown, no lustrous
Achene, Ovate

shape

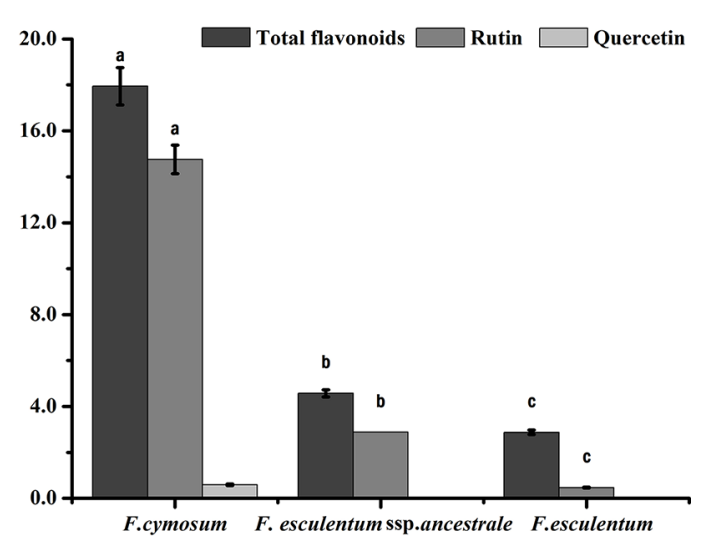

FIGURE 2 | Flavonoids analysis of $F$. esculentum ssp. ancestrale, $F$. cymosum and $F$. esculentum. Error bars represent the standard deviation from three biological replicates determined by a Studentt's t test $(P<0.01)$.

when compared with cp genome of $F$. esculentum it also displayed a typical quadripartite structure including a pair of inverted repeat region 2 IRR $(30,687 \mathrm{bp})$ separated by the LSC $(84,892 \mathrm{bp})$ and small single copy SSC (13,334 bp) regions (Figure 4, Table 1).

Gene annotation studies revealed that the cp genome of $F$. esculentum ssp. ancestrale contains 113 unique genes (Table 3), of which 79 are protein-coding (69.9\%), 30 are transfer RNAs (26.6\%), and 4 are ribosomal RNAs (3.5\%). Further, the functional analysis divided the 113 genes into three categories including 60 transcription and translation regulating genes (53.0\%), 47 genes related to photosynthesis (41.6\%) and 6 genes with unknown function (5.4\%). Half of the transcription and translation regulating genes are the transfer RNA genes and most of the Photosynthesis related genes belong to the photosystem II (31.9\%) and NADPH dehydrogenase (23.4\%).
In total, we found 17 intron-containing genes, including fifteen genes contain one intron, and two genes (ycf3 and clpP) contain two introns. Rps12 is a specific trans-shear gene with its 5' exon in the LSC region and the 3' exon in the IR region (Table 3).

The comparative account of $\mathrm{cp}$ genome and gene annotation studies confirmed that $F$. esculentum ssp. ancestrale is phylogenetically very close to F. esculentum. This was further confirmed by alignment analysis of five buckwheat, where divergence levels of $c p$ genomes were found very low (Figure 5). Overall, the comparative account of cp genomes, revealed that $F$. esculentum ssp. ancestrale was highly similar to F. esculentum, and the chloroplast genome sequence of five buckwheat species was highly conserved.

\section{SSR Polymorphism in the cp Genomes of $F$. esculentum ssp. ancestrale, $F$. cymosum and $F$. esculentum}

The results revealed a total of 44,35 , and 41 SSRs in the $F$. esculentum ssp. ancestrale, F. cymosum, and F. esculentum cp genomes, respectively. An overview of $\mathrm{cp}$ genomes revealed that most of these SSRs were distributed in LSC followed by IR and SSC regions in all the three species (Figure 6A). While comparing the cp genomes of three Fagopyrum species, it was observed that $F$. esculentum ssp. ancestrale shares 27 and 31 identical SSR sequences with $F$. cymosum and $F$. esculentum, respectively. The most abundant SSRs motifs were mononucleotides, accounting for about 79.5, 80, and $78 \%$ of the SSRs motifs in F. esculentum ssp. ancestrale, F. cymosum and F. esculentum, respectively. Meanwhile, in all the three Fagopyrum species, the SSRs with A/T base repeat were significantly more than the G/C repeat. SSR types of $F$. cymosum and F. tataricum were similar, while the repeat types of $F$. esculentum, F. luojishanense, and F. esculentum ssp. ancestrale were closer, all containing the G/C type of SSR 

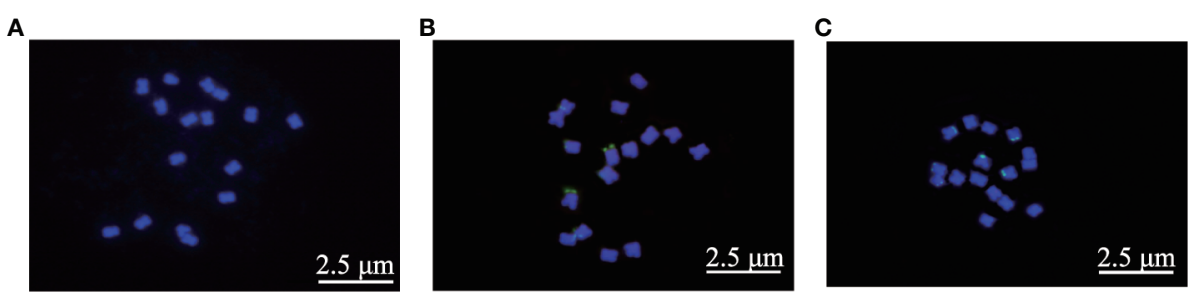

FIGURE 3 | Cytogenetic analysis of F. cymosum (A), F. esculentum ssp. ancestrale (B), and F. esculentum (C). The 25 s fluorescent mark showed that there were 6 fluorescently named chromosome closes in both F. esculentum and F. esculentum ssp. ancestrale. At least three biological replicates of (A-C) were performed with the same results.

repeat sequences (Figure 6B). F. esculentum and F. esculentum ssp. ancestrale have similar type of SSR; Similar between $F$. cymosum and F. tarticum; F. luojishanense is a wild species and in the type of represents composite SSR number is the least compared to the others (Figure 6C). These results indicated that F. esculentum ssp. ancestrale was closer to F. esculentum than F. cymosum.

\section{Phylogenetic Analysis of $F$. esculentum} ssp. ancestrale, F. cymosum and F. esculentum Based on cp Genome

Comprehensive chloroplast genome data was utilized to construct a phylogenic tree to clarify the phylogenetic relationship between F. esculentum ssp. ancestrale, F. cymosum

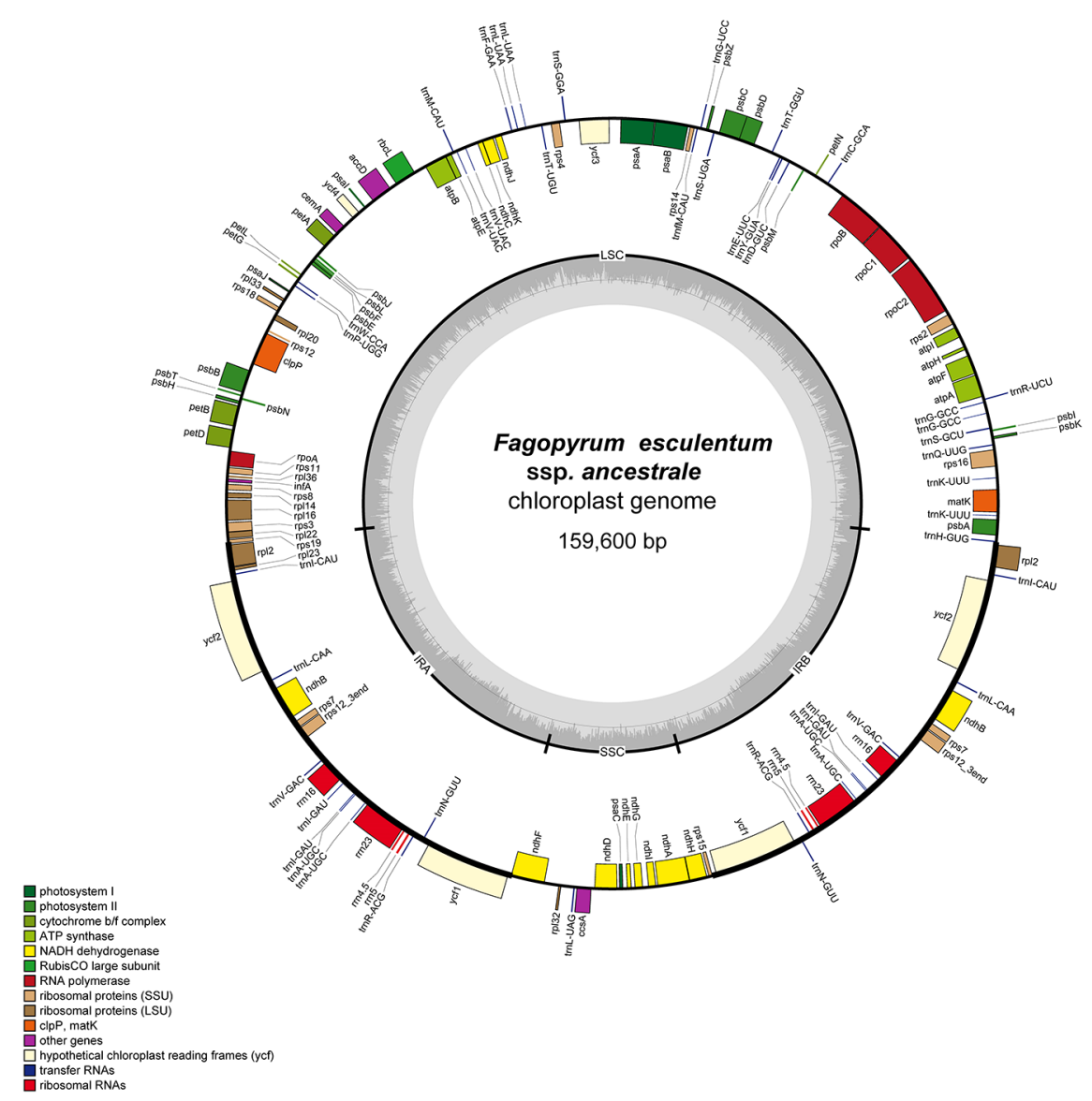

FIGURE 4 | Gene map of $F$. esculentum ssp. ancestrale chloroplast genome. The genes shown outside of the circle are transcribed clockwise, while those inside are counter clockwise. 
TABLE 3 | Gene annotation and classification of chloroplast genome.

\section{Gene category Group of gene}

\section{Name of gene}

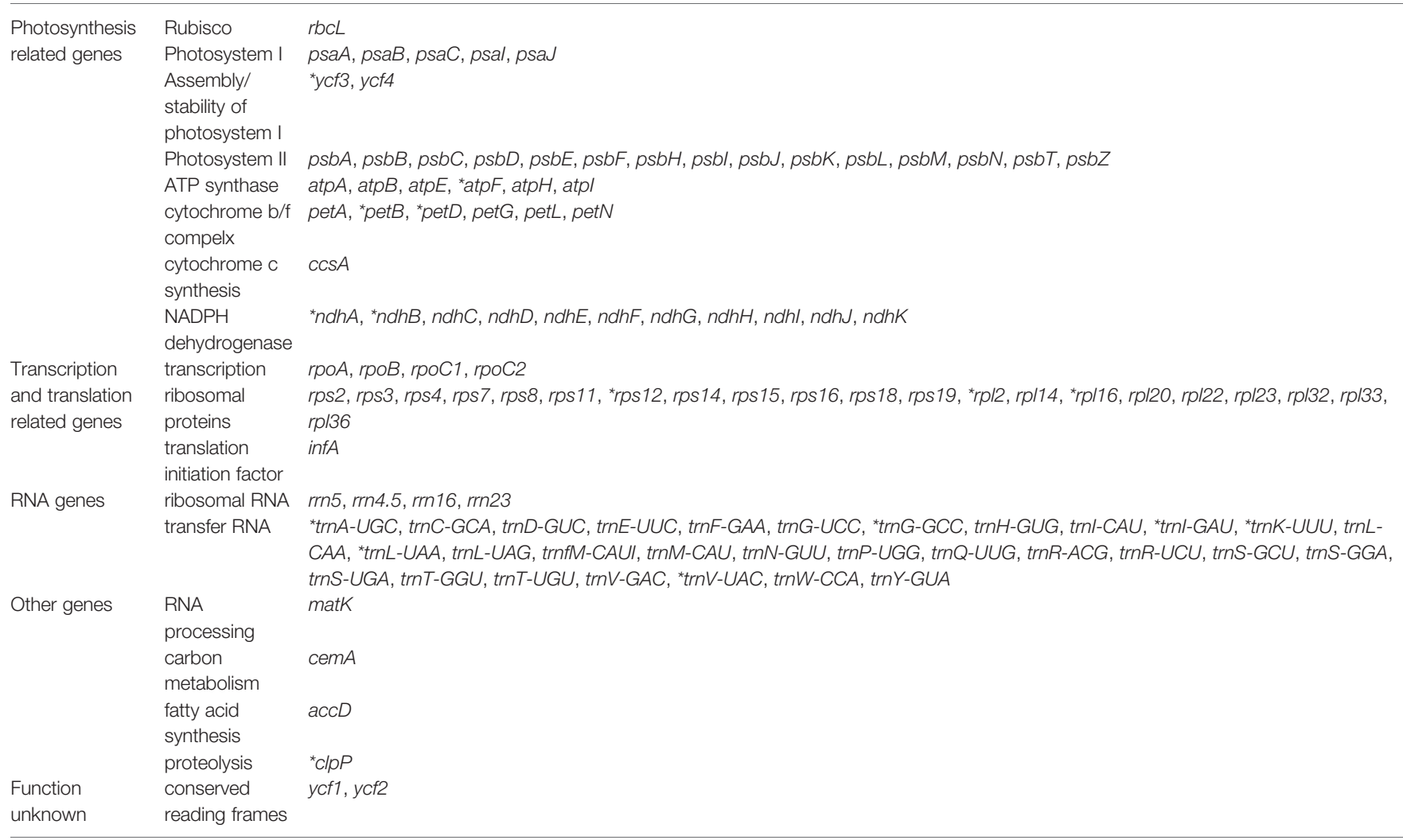

Intron-containing genes are marked by asterisks $\left(^{*}\right)$.

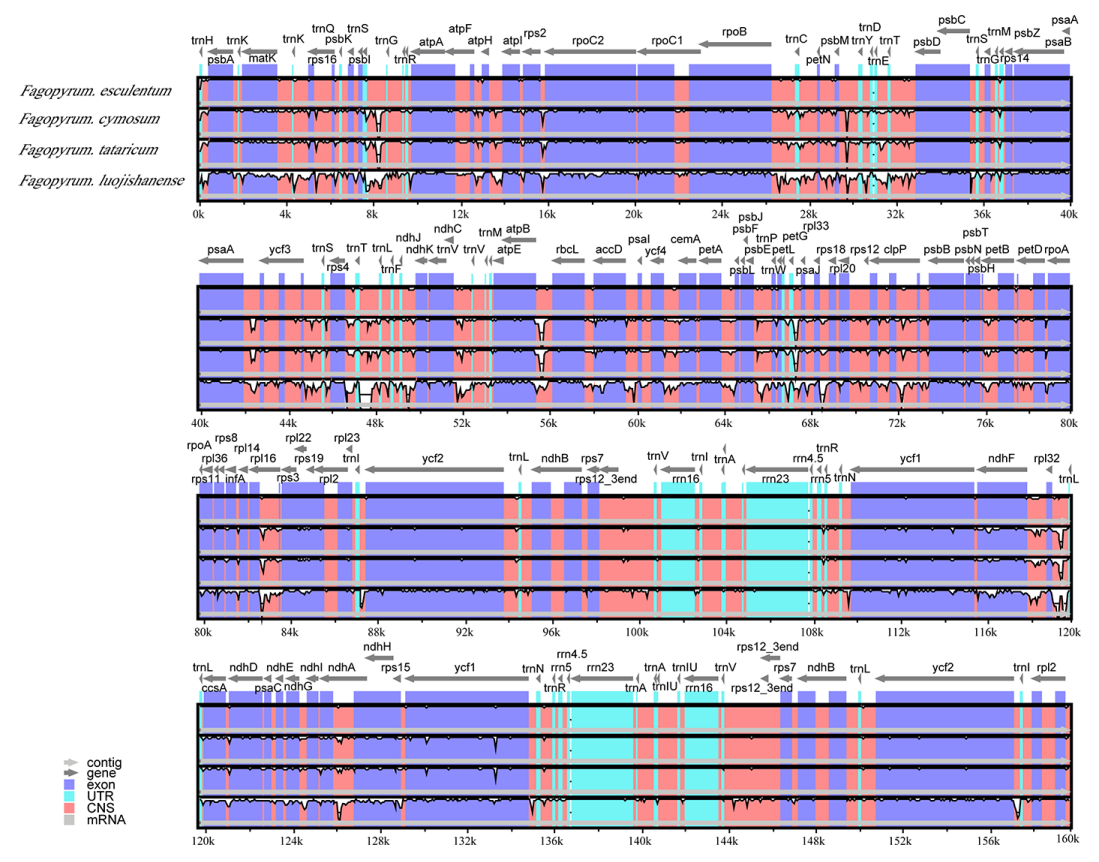

FIGURE 5 | Sequence identity plots of five Fagopyrum chloroplast genomes by using mVISTA. The y-axis represents identity ranging from 50 to $100 \%$. 


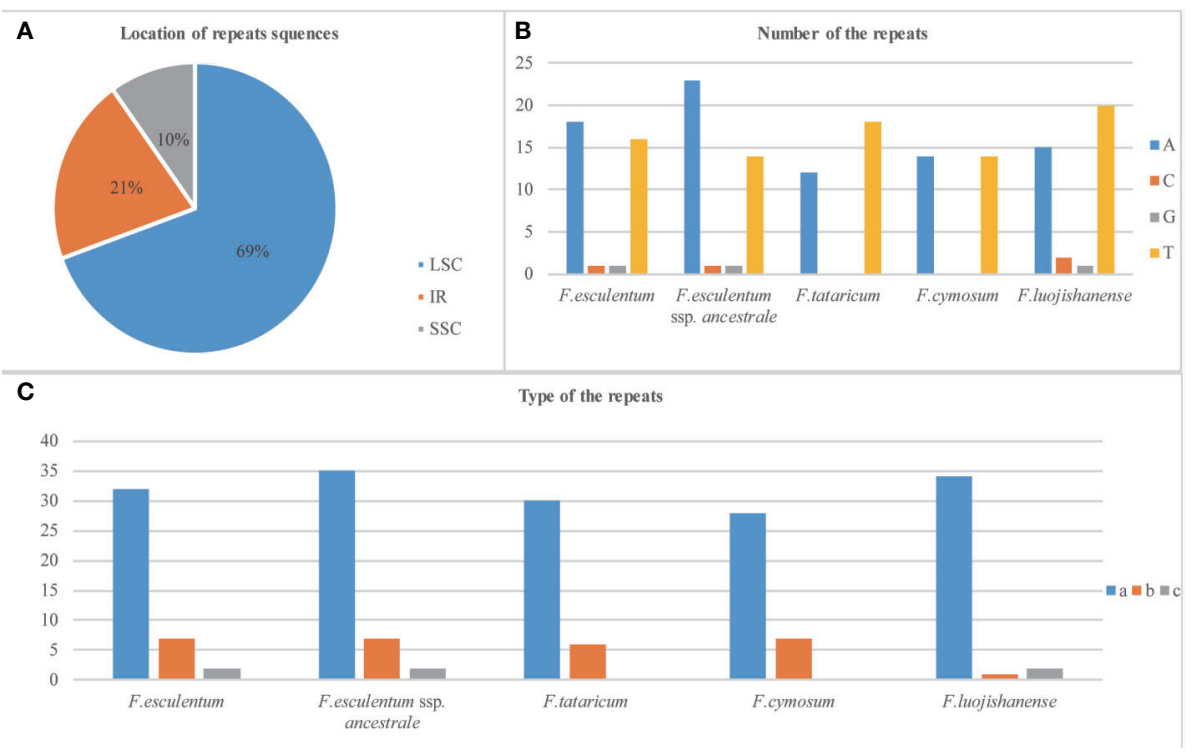

FIGURE 6 | Overview of SSRs in the chloroplast genomes of five Fagopyrum species. (A) Distribution of SSRs in LSC, IR and SSC. (B) Number of nucleic acids in SSRs of five Fagopyrum species. (C) Type of the SSRs of five Fagopyrum species. a represents single base SSR, b represents composite SSR, c represents two base SSR.

and F. esculentum (Figure 7). Along with five chloroplast genome Fagopyrum species, 4 out groups (Table S3) species were used to construct the phylogenic tree, which belongs to other genera in Polygonaceae family. based on these gene data a phylogenetic tree was constructed by using Three different methods i.e. $\mathrm{MP} / \mathrm{MB} / \mathrm{ML}$. The findings signify that all the Fagopyrum species grouped together with very high internal resolution, and other four Polygonaceae species were gathering as the other cluster, Further the tree topology revealed that $F$. esculentum ssp. ancestrale grouped with $F$. esculentum with a high bootstrap score (Figure 7). To further investigate the evolutionary relationships, phylogenetic tree of all the 23 Fagopyrum species was constructed based on two chloroplast gene sequences (mat K and trnhH-psbA) and one nuclear gene sequence ITS). A perusal of tree topology based on ITS (Figure S2), matK (Figure S3), and $t r n h H-p s b A$ (Figure S4) revealed that $F$. esculentum ssp. ancestrale along with three cultivated species (F. esculentum, F. tataricum and F. cymosum) formed a single clade and delineated from rest of the buckwheat species. Further results showed that the F. esculentum ssp. ancestrale and F. esculentum grouped together in a sub-cluster revealing high homology and close hereditary relationship between the two species.

To further investigate the phylogenetic relationship among $F$. cymosum, F. esculentum ssp. ancestrale, and F. esculentum, PCR amplification using SSR markers BM469, BM463, and BM517 (Shi et al., 2015) were performed. SSR analysis revealed that $F$. esculentum ssp. ancestrale exhibits co-dominance with the bands amplified by $F$. cymosum and F. esculentum (Figure S5), supporting our hypothesis about the existence of a hybrid species (F. esculentum ssp. ancestrale) between the evolutionary route from $F$. cymosum to F. esculentum.

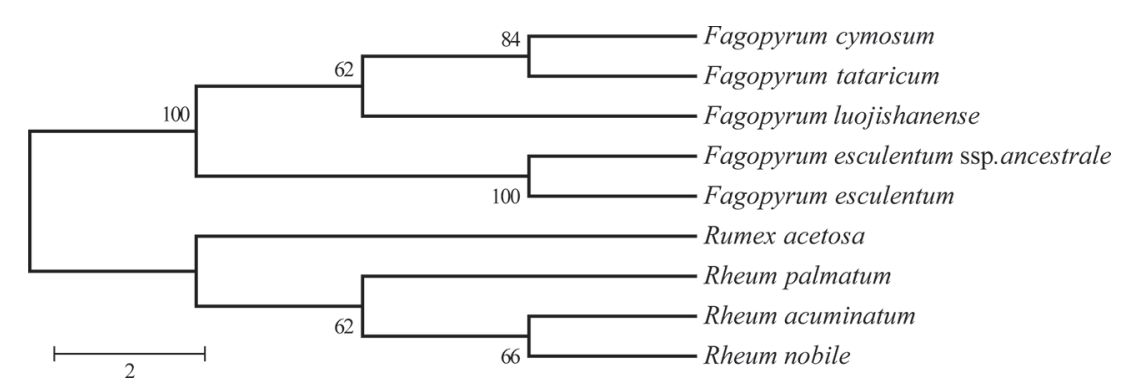

FIGURE 7 | Phylogenetic relationships of the five Fagopyrum species inferred from MLanalysis constructed by chloroplast genome. This polygenetic tree was constricted by RAxML and modified by MEGA and Adobe Al. The number on the branch displayed the bootstrap support values. 


\section{DISCUSSION}

The three buckwheat species were characterized by leaf morphology, inflorescence type, flower type diversity, fruit color, and shape (Table 2). F. esculentum ssp. ancestrale was distinguishable to some extent from the two cultivated species on the basis of perianth color and fruit shape (Table 2). At the same time, F. esculentum ssp. ancestrale exhibits many similarities with $F$. esculentum and F.cymosum, especially with growth habit, petal, leaf, inflorescence type, and fruit color. The ambiguous patterns of morphological descriptors in all the three species support our hypothesis that $F$. esculentum ssp. ancestrale is a hybrid species between F. esculentum and F. cymosum. On the other hand, based on gross morphology and growth habit it is still hard to discriminate the three buckwheat species clearly. Furthermore, parallel and convergent evolution of morphological descriptors and strong dependence on environmental factors for full expression limit their utility for establishing phylogenetic links and distinctiveness among the closely related species and their hybrids (Mes et al., 1997). Therefore, determination of evolutionary relationships and distinctiveness among buckwheat species require more reliable tools like biochemical parameter, cp genome analysis, and molecular markers. There are two main advantages of their use in phylogenetic studies: (1) ease of observation and scoring; and (2) they are usually free from genotype-environment interaction. Additionally, speed of analysis, independence from the growth stage, location, season, and agronomy give them high value in phylogenetic analysis. These attributes which have been tested and confirmed, call for the consideration that biochemical parameters (flavonoids pattern), comparative $\mathrm{cp}$ genome analysis and molecular markers in combination with morphological descriptors will provide concrete genetic data for deducing phylogenetic link of three buckwheat species in our study.

\section{Flavonoids Concentration Established Phylogenetic Relationships Between Fagopyrum Species}

Quantifying secondary metabolites have been used as a reliable method for species identification and determination of phylogenetic relationships between the related species of many plant genera (Bate-Smith and Richens, 1973; Stuessy and Crawford, 1983; Harris, 2009). For instance, the pattern of 21 flavonoids easily differentiated the 14 species of genus crambe offamily cruciferae and also provided hints on the possible phylogenetic relationship between the species (Aguinagalde and Gomez-Campo, 1984). Recently, Zhuang and Tripp (2017) established a correlation between flavonoids pattern, phylogenetic relationships, and latitudinal spread of different species of the Neotropical genus Ruellia. These findings indicated that evolutionary history of different species within a genus is closely related to flavonoids pattern. In this study, we used total flavonoids, rutin and quercetin content for investigating the phylogenetic relationship of $F$. esculentum ssp. ancestrale with $F$. esculentum and F. cymosum. The total flavonoids content for $F$. esculentum ssp. ancestrale observed in the present study $(5.0 \mathrm{mg} / \mathrm{g})$ was considerably low than the total flavonoids content $(13.0 \mathrm{mg} / \mathrm{g})$ observed for $F$. cymosum, but very close to the total flavonoids content of (3.0 $\mathrm{mg} / 100 \mathrm{~g}$ ) reported for F. esculentum. The results have demonstrated the strong correspondence of evolutionary interpretations of these Fagopryum species flavonoids, which also supported our hypothesis of the phylogenetic status of $F$. esculentum ssp. ancestrale.

\section{Karyotype Analysis Revealed Close Genetic Proximity Between F. esculentum ssp. ancestrale and $F$. esculentum}

Karyotype characterization is one of the most reliable methods for species differentiation and establishment of evolutionary relationship between different species of genus Fagopyrum. However, karyotype studies of Fagopyrum species through molecular cytogenetics are not thoroughly investigated and most of the findings were focused on traditional methods of determining the number and size of chromosomes (Neethirajan et al., 2011; Wang et al., 2017a). The diploid genomic constitution with 16 chromosomes of common buckwheat was first recognized by Taylor (1925). On the basis of morphology, most of the chromosomes of genus Fagopyrum are reported to be metacentric and no telocentric or acrocentric chromosomes have been perceived in any of the species (Zhou et al., 2012). Here, we expand this information with the results of FISH and rDNA analysis and demonstrate an upgraded methodology for karyotype analysis in buckwheat. In our study, the karyotype of $F$. cymosum $(2 \mathrm{n}=2 \mathrm{x}=16)$ was reported to be with fifteen metacentric and one submetacentric chromosomes whereas, $F$. esculentum ssp. ancestrale $(2 \mathrm{n}=2 \mathrm{x}=16)$ and F. esculentum $(2 \mathrm{n}=2 \mathrm{x}=16)$ were represented by all the 16 metacentric chromosomes. Furthermore, the observed range of arm ratio (1.033-1.563) and proportion of longest to the shortest chromosome (1.967) for F. esculentum ssp. ancestrale were closely related to the arm ratio (1.119-1.406) and proportion of longest to the shortest chromosome (2.267) observed in F. esculentum. According to the karyotype classification of genomes (Stebbins, 1971), F. cymosum and F. esculentum ssp. ancestrale were appointed to class $1 \mathrm{~A}$, and $F$. esculentum was assigned to the class $1 \mathrm{~B}$ karyoptype. Moreover, the 25s fluorescent mark revealed that there were six similar fluorescently labeled chromosomes in both F. esculentum and $F$. esculentum ssp. ancestrale, which were not found in the F. cymosum. Additionally, no microsatellites were observed in any of the chromosomes in F. esculentum ssp. ancestrale and $F$. esculentum. These similarities observed in chromosomal studies of F. esculentum ssp. ancestrale and F. esculentum provided strong evidence that they are genetically related to each other.

\section{Comparative Plastid Genomics Established Phylogenetic Link of $F$. esculentum ssp. ancestrale With $F$. esculentum and $F$. cymosum}

The comparative analysis of extensively conserved chloroplast genomes has been utilized as a powerful molecular phylogenetic tool to establish evolutionary links between related species of many plant genera including Fagopyrum (Ohsako and Ohnishi, 2000; Zhou et al., 2014; Wang et al., 2017b). In general, chloroplast genomes are circular in shape ranging from 159 to $265 \mathrm{~kb}$ in length and comprised of LSC, SSC, and two copies of 
IR regions (Jansen and Ruhlman, 2012). In our study, chloroplast genomes of F. esculentum ssp. ancestrale, F. cymosum and F. esculentum were represented by a typical circular structure, which consisted of two copies of IR regions separated by the LSC and SSC region. The results highlighted that both IR regions have lower sequence divergence than LSC and SSC regions like in many other plant species, which is probably due to the gene conversion between IR sequences (Khakhlova and Bock, 2006).

According to their function, $\mathrm{cp}$ genes were divided into three categories, the first set of genes was related to transcription and translation, the second set was related to photosynthesis, the third set have a role in biosynthesis of amino acids, fatty acids as well as some genes with unknown functions. Analysis of our data could identify a total of 17 genes containing introns, 15 of which contain 1 intron and $y c f 3$, and $c l p P$ contains 2 introns. Rps 12 is a specific transshear gene with its 5' exon in the LSC region and the 3 ' exon in the IR region. The comparative plastid genome analysis identified highly variable regions, including trnS-trnG, rpoB-trnC, trnT-psbD, ycf3trnS, trnT-trnL, rbcL-accD, ycf4-cemA, psbE-petL, ndhF-rpl32, and $n d h A$ introns. Sequence divergence in most of these regions located in LSC region have been reported as the molecular marker for phylogenetic relationship analysis in plant genera belonging to family Lauraceae (Song et al., 2015), Leguminosae (Drummond, 2008), Solanaceae (Levin et al., 2006) Lamioideae (Scheen and Albert, 2009), and Polygonaceae (Sanchez et al., 2009). Notably, the high nucleotide diversity reported in $y c f 3-\operatorname{trnS}$ region and $n d h A$ intron in this study is specific to Fagopyrum, which is in agreement with the results of Wang et al. (2017b). Unlike, the findings of Zhou et al. (2014), where highly variable sequence information pertaining to $m a t \mathrm{~K}$ - tranK region was used for phylogeny analysis offour wild species of genus Fagopyrum (F. crispatifolium, F. pugense, F. qiangcai, and $F$. wenchuanense) was found relatively conserved in our study. Overall, abundant molecular markers (SSRs and SNPs) can be generated from these highly variable regions for phylogenetic analysis and species identification in buckwheat.

The co-dominant mode of inheritance, hyper variability and high mutation rate of microsatellites (SSRs) make them potential markers for detecting polymorphism at the population level and phylogenetic relationships among species (Goldstein and Schlötterer, 1999). From our results, the most abundant SSRs in cp genomes were mononucleotide repeats followed by the di and trinucleotides. The numbers of mononucleotide repeats were almost similar in cp genomes of $F$. esculentum ssp.ancestrale, $F$. esculentum and F. cymosum. Furthermore, composite SSRs were reported in cp genome of $F$. esculentum ssp.ancestrale and $F$. esculentum, while they were absent in $F$ tataricum. A comparative account of repeat sequences detected in LSC and SSC regions of $F$. esculentum ssp.ancestrale with F. esculentum and F. cymosum revealed that distribution pattern of majority of repeat sequences was same in all the three species. These observations further confirmed that $F$. esculentum ssp.ancestrale has a close phylogenetic link with F. esculentum and F. cymosum. The PCR amplification pattern of F. cymosum, F. esculentum ssp. ancestrale and F. esculentum using SSR markers BM 469, BM 460, and BM 517 (Figure S5), revealed that F. esculentum ssp. ancestrale exhibits codominance with the loci amplified by $F$. cymosum and $F$. esculentum, so we speculate that Fagopyrum esculentum ssp. ancestrale is a hybrid buckwheat species originated through spontaneous hybridization between $F$. cymosum to $F$. esculentum in their native habitat. Previously, utilizing AFLP, SSR, and allozyme variability, Ohnishi (2009) also established a close genetic and evolutionary link between the populations of common buckwheat and F. esculentum ssp. ancestrale.

\section{CONCLUSIONS}

Studies based on floral and seed morphology, secondary metabolites, chloroplast genome analysis, and SSR banding pattern provided supporting evidence in favor of the hypothesis that $F$. esculentum ssp. ancestrale is originated by spontaneous hybridization between $F$. cymosum to F. esculentum under natural conditions.

\section{DATA AVAILABILITY STATEMENT}

The datasets presented in this study can be found in online repositories. The names of the repository/repositories and accession number(s) can be found in the article/ Supplementary Material.

\section{AUTHOR CONTRIBUTIONS}

CC, YF, YT and RJ performed the experiments. KZ and MY analyzed sequencing data. MZ, DJ, and VM designed the research. DCJ, CC, and MZ wrote and revised the manuscript. All authors contributed to the article and approved the submitted version. There was no involvement of the funding providers in designing the study; collecting, analyzing, or interpreting the data; or deciding to submit the paper for publication. The authors have no conflict of interest affecting this paper.

\section{FUNDING}

This work was supported by the National Key R\&D Program of China (2017YFE0117600), the European Union Horizon 2020 project ECOBREED (771367), the National Natural Science Foundation of China (31871536), the Hunan Provincial Natural Science Foundation of China (2016JJ1010) and the Young Talent Supporting Plan of The Crop Science Society of China (2017QNRC182).

\section{SUPPLEMENTARY MATERIAL}

The Supplementary Material for this article can be found online at: https://www.frontiersin.org/articles/10.3389/fpls.2020.01073/ full\#supplementary-material 


\section{REFERENCES}

Aguinagalde, I., and Gomez-Campo, C. (1984). The phylogenetic significance of flavonoids in Crambe L. (Cruciferae). Bot. J. Linn. Soc. 89, 277-288. doi: 10.1111/j.1095-8339.1984.tb02200.x

Bankevich, A., Nurk, S., Antipov, D., Gurevich, A. A., Dvorkin, M., Kulikov, A. S., et al. (2012). SPAdes: a new genome assembly algorithm and its applications to single-cell sequencing. J. Comput. Biol. 19, 455-477. doi: 10.1089/ cmb.2012.0021

Bate-Smith, E., and Richens, R. (1973). Flavonoid chemistry and taxonomy in Ulmus. Biochem. Syst. Ecol. 1, 141-146. doi: 10.1016/0305-1978(73)90004-5

Beier, S., Thiel, T., Münch, T., Scholz, U., and Mascher, M. (2017). MISA-web: a web server for microsatellite prediction. Bioinformatics 33, 2583-2585. doi: 10.1093/bioinformatics/btx198

Cho, K. S., Yun, B. K., Yoon, Y. H., Hong, S. Y., Mekapogu, M., Kim, K. H., et al. (2015). Complete chloroplast genome sequence of tartary buckwheat (Fagopyrum tataricum) and comparative analysis with common buckwheat (F. esculentum). PloS One 10, e0125332. doi: 10.1371/journal.pone.0125332

Drummond, C. S. (2008). Diversifcation of Lupinus (Leguminosae) in the western New World: derived evolution of perennial life history and colonization of montane habitats. Mol. Phylogenet. Evol. 48, 408-421. doi: 10.1016/ j.ympev.2008.03.009

Frazer, K. A., Pachter, L., Poliakov, A., Rubin, E. M., and Dubchak, I. (2004). VISTA: computational tools for comparative genomics. Nucleic Acids Res. 32, W273-W279. doi: 10.1093/nar/gkh458

Goldstein, D., and Schlötterer, C. (1999). Microsatellites: evolution and applications (New York: Oxford University Press).

Harris, E. S. (2009). Phylogenetic and environmental lability of flavonoids in a medicinal moss. Biochem. Syst. Ecol. 37, 180-192. doi: 10.1016/j.bse.2009.02.004

Hsiao, C., Wang, R. R-C., and Dewey, D. R. (1986). Karyotype analysis and genome relationships of 22 diploid species in the tribe Triticeae. Can. J. Genet. Cytol. 28, 109-120. doi: 10.1139/g86-015

Jansen, R. K., and Ruhlman, T. A. (2012). Plastid genomes of seed plants. Genomics of chloroplasts and mitochondria (Dordrecht: Springer) 35, 103126. doi: 10.1007/978-94-007-2920-9_5

Jenkins, G., and Hasterok, R. (2007). BAC'landing'on chromosomes of Brachypodium distachyon for comparative genome alignment. Nat. Protoc. 2, 88-98. doi: 10.1038/nprot.2006.490

Joshi, D. C., Zhang, K., Wang, C., Chandora, R., Khurshid, M., Li, J., et al. (2020). Strategic enhancement of genetic gain for nutraceutical development in buckwheat, A genomics-driven perspective. Biotechnol. Adv. 39, 107479. doi: 10.1016/j.biotechadv.2019.107479

Kadyrova, G., Ryzhova, N., and Kochieva, E. (2010). Phylogenetic relationships among Fagopyrum species based on analysis of the nad1 Gene b/c intron. Moscow. Univ. Biol. Sci. Bull. 65, 161-163. doi: 10.3103/\$0096392510040103

Katoh, K., and Standley, D. M. (2013). MAFFT multiple sequence alignment software version 7: improvements in performance and usability. Mol. Biol. Evol. 30, 772-780. doi: 10.1093/molbev/mst010

Kearse, M., Moir, R., Wilson, A., Stones-Havas, S., Cheung, M., Sturrock, S., et al. (2012). Geneious Basic: an integrated and extendable desktop software platform for the organization and analysis of sequence data. Bioinformatics 28, 1647-1649. doi: 10.1093/bioinformatics/bts199

Khakhlova, O., and Bock, R. (2006). Elimination of deleterious mutations in plastid genomes by gene conversion. Plant J. 46, 85-94. doi: 10.1111/j.1365313X.2006.02673.x

Levan, A., Fredga, K., and Sandberg, A. A. (1964). Nomenclature for centromeric position on chromosomes. Hereditas 52, 201-220. doi: 10.1111/j.16015223.1964.tb01953.x

Levin, R. A., Myers, N. R., and Bohs, L. (2006). Phylogenetic relationships among the "spiny solanums" (Solanum subgenus Leptostemonum, Solanaceae). Am. J. Bot. 93, 157-169. doi: 10.3732/ajb.93.1.157

Li, W. X., Zhu, Z. H., Li, G. Y., Liu, F., Li, Y., and Liu, S. C. (2008). Study on Flavone in Buckwheat Determined by Spectrophotometric Method. J. Plant Genet. Resour. 9 (4), 502-505. doi: 10.13430/j.cnki.jpgr.2008.04.010

Li, J., Wang, S., Jing, Y., Wang, L., and Zhou, S. (2013). A modified CTAB protocol for plant DNA extraction. Chin. Bull. Bot. 48, 72-78. doi: 10.3724/ SP.J.1259.2013.00072
Logacheva, M. D., Samigullin, T. H., Dhingra, A., and Penin, A. A. (2008). Comparative chloroplast genomics and phylogenetics of Fagopyrum esculentum ssp. ancestrale- $a$ wild ancestor of cultivated buckwheat. BMC. Plant. Biol. 8, 59. doi: 10.1186/1471-2229-8-59

Lohse, M., Drechsel, O., Kahlau, S., and Bock, R. (2013). OrganellarGenomeDRAW -a suite of tools for generating physical maps of plastid and mitochondrial genomes and visualizing expression data sets. Nucleic Acids Res. 41, W575W581. doi: 10.1093/nar/gkt289

Mes, T. H. M., Wijers, G. J., and Hart, H. T. (1997). Phylogenetic relationships in Monanthes (Crassulaceae) based on morphological, chloroplast and nuclear DNA variation. J. Evol. Biol. 10, 193-216. doi: 10.1007/s000360050018

Neethirajan, S., Hirose, T., Wakayama, J., Tsukamoto, K., Kanahara, H., and Sugiyama, S. (2011). Karyotype Analysis of Buckwheat Using Atomic Force Microscopy. Microsc. Microanal. 17, 572-577. doi: 10.1017/S1431927611000481

Ohnishi, O., and Matsuoka, Y. (1996). Search for the wild ancestor of buckwheat II. Taxonomy of Fagopyrum (Polygonaceae) species based on morphology, isozymes and cpDNA variability. Genes Genet. Syst. 71, 383-390. doi: 10.1266/ggs.71.383

Ohnishi, O. (1991). Discovery of the wild ancestor of common buckwheat. Fagopyrum 11, 5-10.

Ohnishi, O. (1998). Search for the wild ancestor of buckwheat I. Description of new Fagopyrum (Polygonaceae) species and their distribution in China and the Himalayan hills. Fagopyrum 15, 18-28. doi: 10.1007/BF02861199

Ohnishi, O. (2009). On the origin of cultivated common buckwheat based on allozyme analyses of cultivated and wild populations of common buckwheat. Fagopyrum 26, 3-9.

Ohnishi, O. (2016). "Chapter 1: Molecular Taxonomy of the Genus Fagopyrum," in Molecular Breeding and Nutritional Aspects of Buckwheat (London: Elsevier).

Ohsako and Ohnishi (2000). Intra- and interspecific phylogeny of wild Fagopyrum (Polygonaceae) species based on nucleotide sequences of non-coding regions in chloroplast DNA. Am. J. Bot. 87, 573-582. doi: 10.2307/2656601

Sanchez, A., Schuster, T. M., and Kron, K. A. (2009). A Large-Scale Phylogeny of Polygonaceae Based on Molecular Data. Int. J. Plant Sci. 170, 1044-1055. doi: $10.1086 / 605121$

Scheen, A. C., and Albert, V. A. (2009). Molecular phylogenetics of the Leucas group (Lamioideae; Lamiaceae). Syst. Bot. 34, 173-181. doi: 10.1600/036364409787602366

Shi, J., Li, Y., Zhang, Z., Wu, B., and Wang, A. (2015). Genetic diversity of buckwheat and its wild species. J. Plant Genet. Resour. 16, 443-450. doi: 10.13430/ j.cnki.jpgr.2015.03.002

Song, Y., Dong, W., Liu, B., Xu, C., Yao, X., Gao, J., et al. (2015). Comparative analysis of complete chloroplast genome sequences of two tropical trees Machilus yunnanensis and Machilus balansae in the family Lauraceae. Front. Plant Sci. 6, 662. doi: 10.3389/fpls.2015.00662

Stamatakis, A. (2006). RAxML-VI-HPC: maximum likelihood-based phylogenetic analyses with thousands of taxa and mixed models. Bioinformatics 22, 26882690. doi: 10.1093/bioinformatics/btl446

Stebbins, G. L. (1971). Chromosomal evolution in higher plants. London: Edward Amold, 43-46.

Steward, A. N. (1930). The polygoneae of eastern Asia. Contr.Gray Herb. 88, 1-129.

Stuessy, T. F., and Crawford, D. J. (1983). Flavonoids and phylogenetic reconstruction. Plant Syst. Evol. 143, 83-107. doi: 10.1007/BF00984113

Taylor, W. R. (1925). Chromosome constrictions as distinguishing characteristics in plants. Am. J. Bot. 12, 238-244. doi: 10.2307/2435420

Walling, J. G., Zhang, W., and Jiang, J. (2013). Fluorescence in situ hybridization techniques for cytogenetic and genomic analyses. Rice Protocols (Totowa: Springer) 956, 13-27. doi: 10.1007/978-1-62703-194-3_2

Wang, C. L., Li, Z. Q., Ding, M. Q., Tang, Y., Zhu, X. M., and Liu, J. L. (2017a). Fagopyrum longzhoushanense, a new species of Polygonaceae from Sichuan, China. Phytotaxa 291, 73-80 doi: 10.11646/phytotaxa.291.1.7

Wang, C. L., Ding, M. Q., Zou, C. Y., Zhu, X. M., Tang, Y., Zhou, M. L., et al. (2017b). Comparative analysis of four buckwheat species based on morphology and complete chloroplast genome sequences. Sci. Rep. 7, 6514. doi: 10.1038/ s41598-017-06638-6

Wyman, S. K., Jansen, R. K., and Boore, J. L. (2004). Automatic annotation of organellar genomes with DOGMA. Bioinformatics 20, 3252-3255. doi: 10.1093/bioinformatics/bth352

Zhang, K. X., Logacheva, M. D., Meng, Y., Hu, J., Wan, D., Li, L., et al. (2018). Jasmonate-responsive MYB factors spatially repress rutin biosynthesis in Fagopyrum tataricum. J. Exp. Bot. 69, 1955-1966. doi: 10.1093/jxb/ery032 
Zhou, M. L., Bai, D. Q., Tang, Y., Zhu, X. M., and Shao, J. R. (2012). Genetic diversity of four new species related to southwestern Sichuan buckwheats as revealed by karyotype, ISSR and allozyme characterization. Plant Syst. Evol. 298, 751-759. doi: 10.1007/s00606-011-0586-0

Zhou, M. L., Wang, C. L., Wang, D. Z., Zheng, Y. D., Li, F. L., Zhu, X. M., et al. (2014). Phylogenetic relationship of four new species related to southwestern Sichuan Fagopyrum based on morphological and molecular characterization. Biochem. Syst. Ecol. 57, 403-409. doi: 10.1016/j.bse.2014.09.024

Zhuang, Y., and Tripp, E. A. (2017). The draft genome of Ruellia speciosa (Beautiful Wild Petunia: Acanthaceae). DNA Res. 24, 179-192. doi: 10.1093/ dnares/dsw054
Conflict of Interest: The authors declare that the research was conducted in the absence of any commercial or financial relationships that could be construed as a potential conflict of interest.

Copyright (C) 2020 Cheng, Fan, Tang, Zhang, Joshi, Jha, Janovská, Meglič, Yan and Zhou. This is an open-access article distributed under the terms of the Creative Commons Attribution License (CC BY). The use, distribution or reproduction in other forums is permitted, provided the original author(s) and the copyright owner(s) are credited and that the original publication in this journal is cited, in accordance with accepted academic practice. No use, distribution or reproduction is permitted which does not comply with these terms. 